\title{
Assessment of a Multipurpose Solution by Video Gamers and Non-gamers
}

\author{
Marjorie Jeandervin Rah ${ }^{1, *}$, William Thomas Reindel ${ }^{1}$, Cynthia Denise Adams ${ }^{2}$, \\ Melissa Arielle Brostoff ${ }^{2}$, Lisbeth Anne Wilson ${ }^{3}$, Howard Marc Proskin ${ }^{4}$ \\ ${ }^{1}$ Vision Care, Bausch \& Lomb Incorporated, Rochester, USA \\ ${ }^{2}$ Healthcare Insights, Engine, Princeton, USA \\ ${ }^{3}$ Strategic Insights, Bausch Health, Bridgewater, USA \\ ${ }^{4}$ Howard M. Proskin \& Associates, Rochester, USA
}

\section{Email address:}

Marjorie.Rah@bausch.com(M. J. Rah),Bill.Reindel@bausch.com(W. T. Reindel), Cindy.Adams@enginegroup.com(C.D. Adams), Melissa.Brostoff@enginegroup.com(M. A. Brostoff),Beth.Wilson@bauschhealth.com(L. A. Wilson),

hproskin@hmproskin.com (H. M. Proskin)

${ }^{*}$ Corresponding author

\section{To cite this article:}

Marjorie Jeandervin Rah, William Thomas Reindel, Cynthia Denise Adams, Melissa Arielle Brostoff, Lisbeth Anne Wilson, Howard Marc Proskin. Assessment of a Multipurpose Solution by Video Gamers and Non-gamers. International Journal of Ophthalmology \& Visual Science. Vol. 5, No. 1, 2020, pp. 24-30. doi: 10.11648/j.ijovs.20200501.15

Received: November 18, 2019; Accepted: January 17, 2020; Published: January 31, 2020

\begin{abstract}
Purpose: Video gaming is popular worldwide, and its benefits and risks have come to the attention of health care providers across disciplines. Blink rate, blink amplitude, and tear film stability are compromised during dynamic, prolonged use of digital devices, which can result in symptoms of discomfort with contact lens wear. In addition to lens material and design, the choice of lens care cleaning and disinfecting solution can affect wearer satisfaction. The purpose of this evaluation was to compare real world experiences with a hyaluronan-containing contact lens multipurpose solution (MPS) among subjects classified as video gamers and non-gamers. Methods: This study was a prospective, single arm, open label survey of contact lens wearers who used a computer, smartphone, or tablet for a minimum of three hours daily. Contact lens-wearing gamers (subjects who reported that they play electronic games while wearing contact lenses 2-hours or more daily) and non-gamers were provided Biotrue MPS to use for at least seven-days while wearing a fresh pair of their respective habitual contact lenses. Subjects rated performance attributes via an online survey using a 6-point Likert scale. Results: At baseline, gamers were younger than non-gamers, more likely male, spent more time using digital devices daily, and were more likely to report they suffer from eyestrain, sensitive eyes, and red eyes. Both groups rated all queried lens-wearing performance attributes positively; gamers gave higher mean ratings than did non-gamers to 23 of 24 queried attributes, including all four pertaining to long durations of digital device use. After adjusting for both age and gender, significant differences favoring gamers were noted for all 24 attributes. Conclusions: Contact lens wearing gamers and non-gamers rated Biotrue MPS highly based upon various performance attributes. Biotrue MPS remains a good choice for general lens wear, as well as during tear filmchallenging video game play.
\end{abstract}

Keywords: Multipurpose Solution, Hyaluronan, Contact Lens, Video Game, Lens Comfort, Dryness

\section{Introduction}

Gaming technology and use have made significant advancements since William Higinbotham created "Tennis for Two", widely credited as the world's first video game, in $1958[1,2]$. Gaming has grown over the decades; in the
United States (US) alone, consumers spent over $\$ 43$ billion industry-wide in 2018 [3]. The recent growth of selfidentified gamers aged 13 and older is remarkable, increasing from $58 \%$ in 2013 to $66 \%$ in 2018 [4]. Gaming has evolved from a niche amusement to pervasive mainstream entertainment. 
While early gamers played simple games such as virtual tennis using crude gaming systems connected to television sets of the day, today's gamers play multi-sense video games on a variety of platforms, including dedicated video consoles connected to high definition digital televisions or monitors, mobile smartphones and tablets, and laptop or desktop computers [4]. Frequent gamers play games on multiple platforms, including PC $(62 \%$ of frequent gamers $)$, Smartphone (35\%), and dedicated hand-held systems (21\%) [5]. Action games represent one of the top three types of video games that frequent gamers played most often $(30 \%)$ [5].

Gamers comprise a surprisingly diverse population. While the average gamer in 2017 was 35 years of age, 13\% of both male and female gamers were aged 50 and older [6]. There was an equal distribution of female gamers over and under age 35, but younger male gamers (age 35 and under) outnumbered older male gamers by 3: 2. In 2018, male gamers slightly outnumbered female gamers $(54 \%$ versus $46 \%$, respectively) [3]. On average, gamers spend 5-8 hours gaming weekly [4].

Gamers value video gaming as entertainment, but higher incidences of various maladies such as asthenopia and visual abnormalities [7], as well as gaming addiction [8] have been observed as the gaming population has grown. Contact lens performance, specifically during video game play, is discussed anecdotally in various online forums, yet surprisingly little has been reported in peer-reviewed academic journals. However, computer vision syndrome (CVS), a set of eye and vision symptoms (e.g., eyestrain, red eyes, and sensitive eyes) first associated with long-duration viewing of computer monitors $[9,10]$, and more recently with the use of digital devices (e.g., cell phones, tablets, and e-readers) [11, 12] is well documented; video game play may contribute to symptoms of CVS.

Coincident with the widespread adoption of personal computers in the workplace, symptoms of dry eye due to decreased blink frequency, increased rate of tear evaporation, and decreased tear stability were observed in those that used video display terminals (VDTs) frequently [13-15]. Reduced blink amplitude and tear film disruption during video game play were reported subsequently [16]. Blink rate is reduced during challenging cognitive and visual tasks [17, 18], and incomplete blink frequency increases when reading from a computer screen relative to hard copy $[19,20]$. The effect of cognitive demand upon blinking may be greater than the effect the display medium has on blinking [21]. Nonetheless, reduced blink rate and decreased blink completeness during play of video games, particularly the action games and competitive multi-player games popular with today's gamers are affected both by the challenging cognitive demand and the digital display itself. Contact lens wear may further challenge the tear film during video display viewing. In one study of adapted habitual contact lens-wearers, the average blink rate when not wearing lenses decreased from 19 blinks per minute while listening to music down to 6.2 blinks per minute while playing a video game [22]. However, subjects blinked less completely when playing the game regardless of lens wear, which may be a significant contributor to CVS symptoms [21, 23].

Over the past decade, contact lens and lens care manufacturers have made significant advancements in contact lens materials and lens care products to improve the wearing experience of their users. The choice of lens care cleaning and disinfecting solution can affect wearer satisfaction $[24,25]$. This is attributed, at least in part, to the presence of wetting and comfort agents in the multi-purpose solutions (MPS)s [24, 26]. Typically, an MPS contains one or more demulcent and surfactant for lens cleaning, conditioning, and comfort [26]. Biotrue MPS contains hyaluronan (HA) as a comfort agent and poloxamine 1107 as a surfactant, which facilitates both lens cleaning and lens wetting [27].

Biotrue MPS has been used by millions of contact lens wearers throughout the world for nearly a decade, although it has not been studied specifically in a population of gamers. A study evaluating whether the use of Biotrue MPS could significantly reduce the likelihood that patients drop out of using daily wear contact lenses showed a reduced propensity of discontinuing lens wear following use of Biotrue MPS [25]. Of the 93 subjects most likely to drop out of lens wear, $90 \%$ reduced their likelihood to discontinue contact lens wear after using Biotrue MPS for 2 weeks. Further, of 73 study participants interviewed six months after completion of the study, 93\% were still wearing contact lenses at least once per week. In a separate study [28], 90\% of 291 subjects who evaluated Biotrue MPS blindly with their respective habitual contact lenses rated the MPS positively, but digital video screen use and game play habits were not considered in either study.

In the absence of literature describing contact lens performance during video game play, eye care practitioners (ECPs) rely upon real-world, patient experience of contact lenses and lens care solutions prescribed by peers. These real world studies help evaluate product performance in both broader patient populations and special patient subsets and are becoming more common in published literature [29-34]. The US Food and Drug Administration (FDA) recognizes that such data provide insights into clinical outcomes and recently issued guidance for device manufacturers [35]. The purpose of this real world evaluation was to compare populations of contact lens wearing gamers and non-gamers and their real world experiences using Biotrue MPS.

\section{Materials and Methods}

\subsection{Subject Recruitment}

Contact lens wearers were recruited to use Biotrue MPS with their habitual lenses for seven-days, then complete an online survey regarding their experience. Inclusion criteria were: 1. Eighteen-years of age or older, 2. Habitual wear of 
frequent replacement soft contact lenses (excluded if replaced lenses daily), 3. Able to identify habitual lens brand and disinfectant solution brand used, 4. No history of allergy to contact lens disinfecting solutions, 5. Frequent lens wear (minimum $3 \mathrm{hr}$ per day and 4 days per week), and 6. Spend a minimum of 3 hours daily using a computer, smartphone or tablet. All participants completed an electronic consent document prior to participation in the survey.

\subsection{Subject Gamers and Non-gamers}

Participating subjects were segregated into two groups for data analysis purposes, gamers and non-gamers. Gamers were defined as those subjects who answered that they frequently play computer or video games and play electronic games (on any device or system) while wearing contact lenses 2-hours or more daily.

Subjects were provided one bottle of Biotrue MPS in its current packaging by mail to clean, condition, and disinfect a new pair of their respective habitual lenses each day for at least seven days. After using Biotrue MPS for the seven-day period, participants were contacted and asked to complete a 5-minute online survey to rate various MPS performance characteristics.

\subsection{Statistical Analysis}

Subjects answered each performance attribute statement using a 6-point Likert scale, which included three scores indicating agreement (strongly agree, agree, or slightly agree) and three indicating disagreement (strongly disagree, disagree, or slightly disagree). Quantitative agreement scores were assigned to each subject's responses, using the scale $6=$ strongly agree; $5=$ agree; 4 $=$ slightly agree; $3=$ slightly disagree; 2 = disagree; and 1 $=$ strongly disagree.

For each performance attribute, the percentage of subjects in each group that indicated agreement was tabulated, and a chi-squared test was performed to compare these percentages between the groups. Additionally, the quantitative agreement scores were summarized for each group, and the mean scores were compared between the groups using a t-test. To investigate the potential impact of demographic factors on this comparison, the groups were compared using an analysis of covariance (ANCOVA), which adjusted for age and gender. A level of significance of $p<0.05$ was used for all statistical hypothesis tests.

\section{Results}

\subsection{Subject Demographics}

A total of 581 subjects successfully completed the evaluation (i.e., used Biotrue MPS with a fresh pair of their habitual lenses over a seven-day period and answered all survey questions). Of these, 176 were gamers and 405 non-gamers. At baseline, gamers were younger than nongamers $(36.5 \pm 11.0$ versus $39.7 \pm 11.9$ years of age, respectively; $\mathrm{p}=0.002)$, more likely to be male $(47.7 \%$ versus $28.4 \%$, respectively, $\mathrm{p}<0.001)$, and spent more time daily using digital devices $(8.1 \pm 3.6$ versus $7.3 \pm 3.1$ hours, respectively; $\mathrm{p}=0.007)$. Additionally, gamers at baseline, were more likely than non-gamers to report that they experience eyestrain $(42.6 \%$ versus $26.2 \%$, respectively; $\mathrm{p}<0.001)$, sensitive eyes $(42.6 \%$ versus $31.4 \%$, respectively; $p=0.009)$, and red eyes $(30.1 \%$ versus $19.3 \%$, respectively; $\mathrm{p}=0.004$ ).

\subsection{Subject Evaluation of Biotrue MPS}

After seven-days use of Biotrue MPS with their habitual lenses, subjects were asked to agree or disagree with the attribute statements regarding the MPS listed in Tables 1 and 2. Subjects chose from three levels of agreement or disagreement, and the proportion of subjects that agreed (i.e., responded strongly agree, agree, or slightly agree) or disagreed (i.e., responded strongly disagree, disagree, or slightly disagree) with each statement were calculated. Subjects from both groups overwhelmingly agreed with all attribute statements, with agreement across all attributes ranging from 95.5 to $99.4 \%$ of gamers versus 93.6 to $99.5 \%$ of non-gamers, respectively. A significantly higher percentage of gamers than non-gamers agreed with four of the attributes, these being: helps prevent contact lens dryness $(p=0.035)$, allows me to forget that I am wearing contact lenses $(p=0.047)$, helps prevent contact lens dryness during long durations of using a computer, tablet or smartphone use $(p=0.045)$, and helps prevent eyes from feeling tired or fatigued during long durations of using a computer, tablet or smartphone use $(p=0.021)$. There was no statistically significant difference between the proportion of gamers and non-gamers with respect to agreement with the remaining 20 attributes.

Mean quantitative agreement scores for gamers and nongamers are presented in Tables 1 (general and temporal wear attributes) and 2 (wear attributes during long durations of computer, tablet, or smartphone use). While both groups overwhelming agreed with all queried attribute statements, comparison of mean scores reveals differences in the levels of agreement between groups. As is indicated in the tables, the mean quantitative agreement score was significantly higher for gamers than for non-gamers for all attributes but one (is comfortable upon lens insertion; $\mathrm{p}=0.057$ ), which favored gamers numerically.

Because the age and gender makeup of the gamers and non-gamers differed, it was of interest to know how groups would compare if adjusted for both age and gender. The results of the ANCOVA analyses indicated minimal differences in significance between the unadjusted p-values (presented in Tables 1 and 2) and the age and gender adjusted p-values. After adjusting for both age and gender, gamers expressed a higher level of agreement with all twelve queried general attributes, all eight temporal attributes, and all four attributes during long duration digital device use (all p < $0.05)$. 
Table 1. Quantitative Agreement Scores (Mean \pm Standard Deviation) of Gamers and Non-gamers for Attributes of Biotrue Contact Lens Solution after SevenDays of Use

\begin{tabular}{|c|c|c|c|}
\hline \multicolumn{4}{|l|}{ General } \\
\hline Attribute & Gamers* $(n=176)$ & Non-gamers* $(n=405)$ & P-value (unadjusted) \\
\hline Feels like my natural tears & $5.41 \pm 0.84$ & $5.22 \pm 0.84$ & $0.015^{1 \S}$ \\
\hline Works like my eyes to keep lenses feeling moist & $5.50 \pm 0.76$ & $5.27 \pm 0.80$ & $0.001^{\top \S}$ \\
\hline Maintains my eyes' naturally healthy balance & $5.44 \pm 0.73$ & $5.26 \pm 0.75$ & $0.008^{1 \S}$ \\
\hline Is naturally balanced so it is easy on my eyes & $5.52 \pm 0.69$ & $5.35 \pm 0.73$ & $0.011^{1 \S}$ \\
\hline Makes contact lens wear easy on my eyes & $5.49 \pm 0.68$ & $5.33 \pm 0.78$ & $0.015^{\top \S}$ \\
\hline Allows me to forget that I am wearing contact lenses & $5.30 \pm 0.85$ & $5.05 \pm 0.99$ & $0.003^{1 \S}$ \\
\hline Makes me less aware I am wearing contact lenses & $5.26 \pm 1.00$ & $5.07 \pm 0.98$ & $0.033^{1 \S}$ \\
\hline Is gentle on my eyes & $5.63 \pm 0.70$ & $5.47 \pm 0.72$ & $0.015^{1 \S}$ \\
\hline Keeps my lenses feeling clean & $5.57 \pm 0.61$ & $5.38 \pm 0.79$ & $0.004^{\top \S}$ \\
\hline Makes my lenses feel like new & $5.35 \pm 0.83$ & $5.18 \pm 0.90$ & $0.030^{\top \S}$ \\
\hline Helps prevent contact lens dryness & $5.59 \pm 0.64$ & $5.23 \pm 0.89$ & $<0.001^{\text {\& }}$ \\
\hline Helps reduce lens dryness to break the cycle of discomfort & $5.47 \pm 0.77$ & $5.22 \pm 0.92$ & $0.002^{\top \S}$ \\
\hline \multicolumn{4}{|l|}{ Temporal } \\
\hline Attribute & Gamers* $(n=176)$ & Non-gamers* $(n=405)$ & P-value (unadjusted) \\
\hline Is comfortable upon lens insertion & $5.63 \pm 0.70$ & $5.51 \pm 0.67$ & $0.057^{\S}$ \\
\hline Helps my lenses stay comfortable throughout the day & $5.54 \pm 0.71$ & $5.37 \pm 0.77$ & $0.012^{1 \S}$ \\
\hline Helps my lenses stay comfortable at the end of the day & $5.45 \pm 0.81$ & $5.27 \pm 0.84$ & $0.015^{1 \S}$ \\
\hline Helps my eyes feel fresh at the end of the day & $5.42 \pm 0.83$ & $5.20 \pm 0.93$ & $0.007^{\top \S}$ \\
\hline Helps my lenses stay moist throughout the day & $5.57 \pm 0.66$ & $5.30 \pm 0.83$ & $<0.001^{1 \S}$ \\
\hline Helps my lenses stay moist at the end of the day & $5.50 \pm 0.81$ & $5.20 \pm 0.91$ & $<0.001^{1 \S}$ \\
\hline Helps lenses stay moist and comfortable all day long & $5.45 \pm 0.75$ & $5.30 \pm 0.85$ & $0.036^{1 \S}$ \\
\hline Helps my lenses stay comfortable when using a digital device for long hours & $5.49 \pm 0.66$ & $5.31 \pm 0.83$ & $0.008^{\top \S}$ \\
\hline
\end{tabular}

*Quantitative agreement scores were assigned to each subject's responses, using the scale $6=$ strongly agree; $5=$ agree; $4=$ slightly agree; $3=$ slightly disagree; 2 = disagree; and 1 = strongly disagree. These scores were quantitatively summarized, and t-tests were utilized to compare the mean scores between groups.

Significantly different at $\mathrm{p}<0.05$.

${ }^{\S}$ Significantly different at $\mathrm{p}<0.05$ after adjusting for age and gender.

Table 2. Quantitative Agreement Scores (Mean \pm Standard Deviation) for Gamers and Non-gamers During Long Durations of Using a Computer, Tablet or Smartphone Use for Attributes of Biotrue Contact Lens Solution after Seven-Days of Use

\begin{tabular}{lll}
\hline Attribute & Gamers* $(\mathbf{n}=\mathbf{1 7 6})$ & Non-gamers* $(\mathbf{n}=\mathbf{4 0 5})$ \\
\hline Helps my lenses stay comfortable throughout the day & $5.60 \pm 0.69$ & $5.32 \pm 0.80$ \\
Helps prevent contact lens dryness & $5.62 \pm 0.61$ & $5.25 \pm 0.86$ \\
Helps lenses stay moist and comfortable all day long & $5.53 \pm 0.70$ & $5.29 \pm 0.83$ \\
Helps prevent eyes from feeling tired or fatigued & $5.36 \pm 0.82$ & $5.05 \pm 0.92$ \\
\hline
\end{tabular}

*Quantitative agreement scores were assigned to each subject's responses, using the scale $6=$ strongly agree; $5=$ agree; $4=$ slightly agree; $3=$ slightly disagree; 2 = disagree; and 1 = strongly disagree. These scores were quantitatively summarized, and t-tests were utilized to compare the mean scores between groups.

'Significantly different at $\mathrm{p}<0.05$.

${ }^{\S}$ Significantly different at $\mathrm{p}<0.05$ after adjusting for age and gender.

\section{Discussion}

Habitual gamers' prolonged daily use of digital devices and frequent action game play requiring intense concentration and consequently suppressing blink rate and blink completeness make this population especially susceptible to suboptimal contact lens wear. Such activities are reported to cause eye and vision symptoms such as eyestrain, red eyes, and sensitive eyes that are similar to if not the same as the CVS symptoms experienced by heavy computer users $[9,10]$. Indeed, in this study a higher fraction of subjects in the gamers group experienced such symptoms at baseline compared with the non-gamers group. Given the potential for reduced blink rate and increased frequency of incomplete blinks during game play, one might expect that differences would be present between gamers and nongamers with respect to aggregate agreement scores. This study was undertaken to compare how populations of gamers and non-gamers perceive Biotrue MPS performance when used with their respective habitual lenses.

Contact lens-wearing gamers and non-gamers alike found that use of Biotrue MPS for seven days resulted in a positive lens wearing experience based upon a number of characteristic performance attributes (agreement range 93.6 $99.5 \%$ across all attributes). A significantly greater fraction of gamers agreed with four of the twenty-four queried attribute statements compared with non-gamers, and eighteen of the remaining twenty attributes favored gamers but were not significantly different between groups. Further, gamers had significantly higher quantitative scores for all queried 
attributes but one, which also favored gamers but was not significantly different. These results are consistent with those of a previous study in which $90 \%$ of habitual contact lens wearers rated the MPS highly [28], as well as another that found $80 \%$ reduced likelihood of discontinuing lens wear following use of Biotrue MPS by patients considering dropping out of daily wear contact lenses [25].

Tear function [36], as well as dry-eye disease and tear meniscus height [37], are inversely correlated with age, and dry eye is more prevalent in women than in age-matched men [38], which suggests that differences in response might be due to age and gender. However, while higher gamer satisfaction could be thought due to that group's younger average age (36.5 versus 39.7 years) and greater likelihood to be male gender ( $47.7 \%$ versus $28.4 \%$ ), adjusting for both age and gender when comparing mean quantitative scores for each attribute refutes this hypothesis. Significant differences were noted in favor of gamers for all adjusted attributes, i.e., the differences between gamers and nongamers became significant for the attribute, "is comfort upon lens insertion," after age and gender adjustment, while differences for all other attributes remained significant, indicating that age and gender had little effect on mean attribute ratings. Despite the challenges faced by gamers, use of Biotrue MPS provided greater benefit to gamers as reflected by significantly greater mean attribute scores compared with non-gamers.

Positive perceptions of Biotrue may reflect the retention of multiple comfort agents in the MPS formulation. An unfavorable consequence of incomplete blinks and reduced blink rate during game play is reduced tear film stability. Surfactants help stabilize the tear film by promoting spreading of the tears across the lens and inhibiting lens dewetting.

Poloxamine 1107 is a non-ionic surfactant included in Biotrue MPS that functions as a wetting/ conditioning agent and facilitates lens cleaning. It is reported to maintain etafilcon A lens wettability over the first four hours of wear and be retained within lenses over the first eight hours [39]. Demulcents also help stabilize the tear film by increasing moisture retention by the lens. The high-molecular-weight (HMW) HA in Biotrue MPS contributes to comfort based upon its unique biological and physical properties [40], including viscoelasticity [41], hydrophilicity [42, 43], hygroscopy (HA is reported to bind 1,000 times its weight in water) [44], and lubricity [43, 45].

Previous studies of Biotrue MPS demonstrated sorption of HA on both traditional and silicone hydrogel contact lenses, and subsequent HA elution into artificial tear fluid in vitro over a 20-hour period [46, 47]. HA was also shown to persist in the tears of contact lens wearers after two-hours wear of senofilcon A lenses that had previously been soaked in Biotrue MPS [48]. Further, the wettability of several contact lens materials was reported to increase after conditioning with Biotrue MPS relative to their respective lens packaging solutions [49]. These findings likely contributed to the enhanced wearing experience of both gamers and non- gamers in the present study.

One limitation of this study was the lack of a control MPS. However, real world, non-randomized, prospective evaluations can indicate important trends that add to the knowledge base and may lead to additional clinical studies. For example, this study found that use of Biotrue MPS leads to high user satisfaction and greater satisfaction among gamers compared with non-gamers.

\section{Conclusions}

Biotrue MPS contains the unique combination of HA and poloxamine 1107, which enhances lens wetting. Contact lens wearing gamers and non-gamers alike rated the MPS highly based upon a set of performance attributes when queried after seven-days use. However, despite being more susceptible to eyestrain, sensitive eyes, and red eyes, gamers using Biotrue MPS reported significantly better lens performance than did non-gamers, notably during long durations of digital device use. As such, ECPs should consider recommending Biotrue MPS to both frequent-gaming patients and patients that use digital devices for long durations daily.

\section{Disclosure}

Authors Rah and Reindel, Wilson are direct employees of Bausch \& Lomb Incorporated. Author Wilson is a direct employee of Bausch Health. Both Engine and Howard M. Proskin \& Associates are paid consultants to Bausch \& Lomb Incorporated.

\section{References}

[1] Higinbotham WA. BNL Tennis Game. Deposition of April 29, 1976. Available from: https://www.bnl.gov/about/docs/Higinbotham_Deposition.pdf. Accessed November 18, 2019.

[2] This Month in Physics History. October 1958: Physicist invents first video game. APS News. 2008; 17 (9): 2. Available from: https://www.aps.org/publications/apsnews/200810/upload/Oct ober-2008-Volume-17-Number-9-Entire-Issue.pdf. Accessed November 18, 2019.

[3] 2019 Essential Facts about the Computer and Video Game Industry. Washington, DC: The Entertainment Software Association (ESA) 2018. Available from: https://www.theesa.com/wp-content/uploads/2019/05/2019Essential-Facts-About-the-Computer-and-Video-GameIndustry.pdf. Accessed November 18, 2019.

[4] Flamberg M. U.S. Games 360 Report: 2018. Nielsen Holdings plc 2018; pp 6-8. Available from: http://www.nielsen.com/us/en/insights/reports/2018/us-games360-report-2018.html. Accessed November 18, 2019.

[5] 2015 Sales, Demographic and Usage Data. Essential Facts about the Computer and Video Game Industry. Washington, DC: The Entertainment Software Association (ESA) 2015. Available from: https:/templatearchive.com/esa-essentialfacts/. Accessed November 18, 2019. 
[6] 2017 Sales, Demographic and Usage Data. Essential Facts about the Computer and Video Game Industry. Washington, DC: The Entertainment Software Association (ESA) 2018. Available from: https://www.theesa.com/wpcontent/uploads/2019/03/ESA_EssentialFacts_2017.pdf. Accessed November 18, 2019.

[7] Rechichi C, De Mojà G, Aragona P. Video game vision syndrome: a new clinical picture in children? [J]. Pediatr Ophthalmol Strabismus. 2017; 54 (6): 346-355.

[8] Wittek CT, Finserås TR, Pallesen S, Mentzoni RA, Hanss D, Griffiths MD, Molde H. Prevalence and predictors of video game addiction: a study based on a national representative sample of Gamers. Int J Ment Health Addict. 2016; 14 (5): 672-686.

[9] Blehm C, Vishnu S, Khattak A, Mitra S, Yee RW. Computer vision syndrome: a review. Surv Ophthalmol. 2005; 50 (3): 253-262.

[10] Rosenfield M. Computer vision syndrome: a review of ocular causes and potential treatments. Ophthalmic Physiol Opt. 2011; 31 (5): 502-515

[11] Thevisioncouncil.org [homepage on the Internet]. Hindsight is 20/20/20: Protect Your Eyes form Digital Devices. 2015 Digital Eye Strain Report. Alexandria, VA: The Vision Council. 2015 [cited July 1, 2019]. Available from: https://www.pcom.ph/sites/default/files/downloads/vc_digitale yestrain_report2015.pdf. Accessed November 18, $201 \overline{9}$.

[12] Porcar E, Pons AM, Lorente A. Visual and ocular effects from the use of flat-panel displays. Int J Ophthalmol. 2016; 9 (6): $881-885$.

[13] Patel S, Henderson R, Bradley L, Galloway B, Hunter L. Effect of visual display unit use on blink rate and tear stability. Optom Vis Sci. 1991; 68 (11): 888-892.

[14] Tsubota K, Nakamori K. Dry eyes and video display terminals. N Engl J Med. 1993; 328 (8): 584

[15] Wolkoff P, Nøjgaard JK, Troiano P, Piccoli B. Eye complaints in the office environment: precorneal tear film integrity influenced by eye blinking efficiency. Occup Environ Med. 2005; 62 (1): 4-12.

[16] Cardona G, García C, Serés C, Vilaseca M, Gispets J. Blink rate, blink amplitude, and tear film integrity during dynamic visual display terminal tasks. Curr Eye Res. 2011; 36 (3): 190197.

[17] Bentivoglio AR, Bressman SB, Cassetta E, Carretta D, Tonali $P$, Albanese A. Analysis of blink rate patterns in normal subjects. Mov Disord. 1997; 12 (6): 1028-1034.

[18] Himebaugh NL, Begley CG, Bradley A, Wilkinson JA. Blinking and tear break-up during four visual tasks. Optom Vis Sci. 2009; 86 (2): E106-114.

[19] Chu CA, Rosenfield M, Portello JK. Blink patterns: reading from a computer screen versus hard copy. Optom Vis Sci. 2014; 91 (3): 297-302.

[20] Argilés M, Cardona G, Pérez-Cabré E, Rodríguez M. Blink rate and incomplete blinks in six different controlled hardcopy and electronic reading conditions. Invest Ophthalmol Vis Sci. 2015; 56 (11): 6679-85.

[21] Rosenfield M, Jahan S, Nunez K, Chan K. Cognitive demand, digital screens and blink rate. Computers Human Behav. 2015; 51A: 403-406.

[22] Jansen ME, Begley CG, Himebaugh NH, Port NL. Effect of contact lens wear and a near task on tear film break-up. Optom Vis Sci. 2010; 87 (5): 350-357.

[23] Portello JK, Rosenfield M, Chu CA. Blink rate, incomplete blinks and computer vision syndrome. Optom Vis Sci. 2013; 90 (5): 482-487.

[24] Yang SN, Tai YC, Sheedy JE, Kinoshita B, Lampa M, Kern JR. Comparative effect of lens care solutions on blink rate, ocular discomfort and visual performance. Ophthalmic Physiol Opt. 2012; 32 (5): 412-20.

[25] Rah MJ, Merchea MM, Doktor MQ. Reducing dropout of contact lens wear with Biotrue multipurpose solution. Clin Ophthalmol. 2014; 8: 293-9.

[26] Jones L. Soft Contact Lens Solutions Review. Part 1: Components of modern care regimens. Optom Pract. 2007; 8 (1): 45-56.

[27] FDA 510 (k) Summary K083757. Bausch \& Lomb BPZO2 MultiPurpose Solution. November 18, 2009. Available from: https://www.accessdata.fda.gov/cdrh_docs/pdf8/k083757.pdf. Accessed November 18, 2019.

[28] Reindel W, Cairns G, Merchea M. Assessment of patient and practitioner satisfaction with Biotrue ${ }^{\mathrm{TM}}$ multi-purpose solution for contact lenses. Cont Lens Anterior Eye. 2010; 33 (Suppl 1): S12-S17.

[29] Rah MJ, Saxon J, Riendel WT. Real-world performance captures the presbyopic opportunity. Cont Lens Spect. 2016; 31 (13SE): 36-40.

[30] Beusterien K, Tsay S, Gholizadeh S, Su Y. Real-world experience with colorectal cancer chemotherapies: patient web forum analysis. Ecancermedicalscience. 2013; 7: 361.

[31] Boeru G, Milanov I, De Robertis F, et al. ExtaviJect ${ }^{\circledR} 30 \mathrm{G}$ device for subcutaneous self-injection of interferon beta- $1 \mathrm{~b}$ for multiple sclerosis: a prospective European study. Med Devices (Auckl). 2013; 6: 175-184

[32] Chaudhuri N, Duck A, Frank R, Holme J, Leonard C. Real world experiences: pirfenidone is well tolerated in patients with idiopathic pulmonary fibrosis. Respir Med. 2014; 108 (1): $224-226$.

[33] Ge JB, Zhang F, Qian JY, Ge L, Liu XB, Zhou J. Six-month clinical outcomes of Firebird $2^{\mathrm{TM}}$ sirolimus-eluting stent implantation in real-world patients with coronary artery diseases. Chin Med J (Engl). 2011; 124 (6): 831-835.

[34] Han YL, Chen JY, Xu B, et al. Real world clinical performance of the zotarolimus eluting coronary stent system in Chinese patients: a prospective, multicenter registry study. Chin Med J (Engl). 2011; 124 (20): 3255-3259.

[35] FDA Guidance Document. Use of Real-World Evidence to Support Regulatory Decision-Making for Medical Devices. Guidance for Industry and Food and Drug Administration. August 31, 2017. Available from: https://www.fda.gov/media/99447/download. Accessed November 18, 2019.

[36] Ozdemir M, Temizdemir H. Age- and gender-related tear function changes in normal population. Eye (Lond). 2010; 24 (1): 79-83. 
[37] Qiu X, Gong L, Sun X, Jin H. Age-related variations of human tear meniscus and diagnosis of dry eye with Fourierdomain anterior segment optical coherence tomography. Cornea. 2011; 30 (5): 543-9.

[38] Farrand KF, Fridman M, Stillman IÖ, Schaumberg DA. Prevalence of diagnosed dry eye disease in the United States among adults aged 18 years and older. Am J Ophthalmol. 2017; 182: 90-98.

[39] Tonge S, Jones L, Goodall S, Tighe B. The ex vivo wettability of soft contact lenses. Curr Eye Res. 2001; 23 (1): 51-9.

[40] Rah M. A review of hyaluronan and its ophthalmic applications. Optometry. 2011; 82 (1): 38-43.

[41] Fakhari A, Berkland C. Applications and emerging trends of hyaluronic acid in tissue engineering, as a dermal filler and in osteoarthritis treatment. Acta Biomater. 2013; 9 (7): 70817092 .

[42] Lerner LE, Schwartz DM, Hwang DG, Howes EL, Stern R. Hyaluronan and CD44 in the human cornea and limbal conjunctiva. Exp Eye Res. 1998; 67 (4): 481-484.

[43] Brown MB, Jones SA. Hyaluronic acid: a unique topical vehicle for the localized delivery of drugs to the skin. [J]. Eur Acad Dermatol Venereol. 2005; 19 (3): 308-318.
[44] Laurent TC. Structure of hyaluronic acid. In: Chemistry and Molecular Biology of the Intercellular Matrix (EA Balazs, Ed.). London and New York: Academic Press; 1970: 703-732.

[45] Winter WT, Arnott S. Hyaluronic acid: the role of divalent cations in conformation and packing. [J]. Mol Biol. 1977; 117 (3): 761-784.

[46] Scheuer CA, Doty K, Liranso T, Burke S. Wetting agent retention and release from hydrogel and silicone hydrogel contact lenses. Invest Ophthalmol Vis Sci. 2011; 52: e-abstract 6487. (Poster presented at Annual Meeting of the Association for Research in Vision and Ophthalmology (ARVO); 5 May 2011; Fort Lauderdale, Florida, USA.) Available from: https://iovs.arvojournals.org/article.aspx?articleid=2358581. Accessed November 18, 2019.

[47] Scheuer CA, Fridman KM, Barniak VL, Burke SE, Venkatesh $\mathrm{S}$. Retention of conditioning agent hyaluronan on hydrogel contact lenses. Cont Lens Anterior Eye. 2010; 33 (Suppl 1): S2-6.

[48] Scheuer CA, Rah MJ, Reindel WT. Increased concentration of hyaluronan in tears after soaking contact lenses in Biotrue multipurpose solution. Clin Ophthalmol. 2016; 10: 1945-1952.

[49] Fagehi RA, Tomlinson A, Manahilov V. Comparative study of soft contact lens wetting in vitro after storage in Biotrue MPS. Cont Lens Anterior Eye. 2012; 35 (Suppl 1): e21. 